\title{
Horizontal Bone Reconstruction on sites with different amounts of native bone: a retrospective study
}

\section{André Antonio PELEGRINE(a) Giuseppe ROMITO(b) \\ Cristina Cunha VILLAR ${ }^{(b)}$ \\ Luís Guilherme Scavone \\ de MACEDO'(a) \\ Marcelo Lucchesi TEIXEIRA(a) \\ Antonio Carlos ALOISE ${ }^{(a)}$ \\ Peter Keyren MOY(c)}

(a) Centro de Pesquisas Odontológicas São Leopoldo Mandic, Campinas, SP, Brazil.

(b) Universidade de São Paulo - USP, School of Dentistry, Department of Stomatology, São Paulo, SP, Brazil.

(c) University of California, Surgical Implant Dentistry Program, Los Angeles, CA, USA.

Declaration of Interests: The authors certify that they have no commercial or associative interest that represents a conflict of interest in connection with the manuscript.

Corresponding Author:

André Antonio Pelegrine

E-mail: pelegrineandre@gmail.com

https://doi.org/10.1590/1807-3107bor-2018.vol32.0021

Submitted: October 16, 2017

Accepted for publication: January 28, 2018

Last revision: February 15, 2018

\begin{abstract}
The lack of guidelines for bone augmentation procedures might compromise decision making in implantology. The objective of this study was to perform a retrospective study to verify the outcomes of horizontal bone reconstruction in implant dentistry with different types of materials and amounts of native bone in the recipient bed to allow for a new guideline for horizontal bone reconstruction. One hundred preoperative CT scans were retrospectively evaluated and categorized in accordance to horizontal bone defects as presence (Group P) or absence (Group A) of cancellous bone in the recipient bed. Different approaches were used to treat the edentulous ridge and the outcomes were defined either as satisfactory or unsatisfactory regarding the possibility of implant placement. The percentage distribution of the patients according to the presence or absence of cancellous bone was 92\% for Group P and 8\% for Group A. In Group P, 98\% of the patients had satisfactory outcomes, and the use of autografts had $100 \%$ of satisfactory outcomes in this group. In Group A, 37.5\% of the patients had satisfactory outcomes, and the use of autografts also yielded $100 \%$ of satisfactory outcomes. The use of allografts and xenografts in Group A had 0\% and 33.3\% of satisfactory outcomes, respectively. Therefore, it seems reasonable to speculate that the presence of cancellous bone might be predictive and predictable when the decision includes bone substitutes. In cases of absence of cancellous bone in the recipient bed, the use of a vitalized graft seems to be mandatory.
\end{abstract}

Keywords: Dental Implantation; Athrophy; Maxilla; Mandible.

\section{Introduction}

Contemporary implant dentistry has existed for decades, always following the guidelines previously set out by Prof. Per Ingvar Branemark. His well-known protocol established that it is necessary to have bone volume, a sterile and biocompatible implant, an atraumatic surgery, implant with primary stability, and lack of functional loading during the healing period for successful osseointegration. ${ }^{1}$ These guidelines were a breakthrough because they allowed for more predictable implantsupported rehabilitations. However, in cases where bone volume is not adequate for implant placement, bone augmentations are required. ${ }^{2}$ Unlike implant placement, bone augmentation procedures lack guidelines that could help the clinician choose the best materials and techniques. 
Autogenous bone, because of its biological properties (i.e., osteoconduction, osteoinduction, and osteogenesis), is still considered the gold standard for bone reconstruction; however, it is associated with morbidity and with some postoperative complications. ${ }^{3,4}$ On the other hand, another alternative for bone reconstruction is the use of bone substitutes, which were described in the literature as having good outcomes in several clinical situations. $5,6,7,8$ Traditionally, these biomaterials are classified according to their origin into allogeneic, xenogeneic, or alloplastic, instead of being classified according to their biological potential. Advances in biomaterials research and the development of new surgical techniques and armamentarium have resulted in an increased use of implants for tooth replacement. The long-term success of dental implants is highly dependent upon the degree of osseointegration in sufficient and healthy bone.

In regard to biological properties, most bone substitute biomaterials are considered only osteoconductive, because they behave like a scaffold that allows the ingrowth of vessels and cells, enabling revascularization and new bone formation. ${ }^{9}$ Despite the possibility of having autografts and osteoconductive bone substitutes, it has been difficult to choose which could be the best choice for different clinical situations. Furthermore, the outcomes of the procedure depend on the anatomical characteristics of the residual bone, which is important to define the best technique to be used. It is well known that the cortical lamellar bone does not have adequate vitality ${ }^{10}$ and that the cancellous compartment has high vitality and huge osteogenic potential, mainly due to the endosteum and to the bone marrow. ${ }^{11}$ Therefore, the hypothesis that there should be some amount of cancellous bone in the recipient bed to indicate the use of an osteoconductive biomaterial seems to be plausible.

The aim of this study was to retrospectively evaluate the outcomes of appositional grafts used to promote horizontal augmentation by using autogenous bone grafting - the biological gold standard - and osteoconductive substitutes (i.e., homogenous, alloplastic, or xenogenous grafts) in different types of recipient beds concerning, mainly, the presence or absence of cancellous bone.

\section{Methodology}

This study was performed by the retrospectively evaluation of 100 patients subjected to implant therapy on previously healed sites (i.e., no immediate implants). A total of 100 patients treated by our group with implants, which should be functional for at least 1 year, were assessed. The previous CT scans of the selected patients were evaluated to determine the need and extension of bone grafts. Free and informed consent was obtained from all patients. Due to the retrospective nature of this study, after analysis by the research ethics committee, the need of a written form was dismissed by the São Leopoldo Mandic Institute and Research Center.

\section{Inclusion criteria}

The CT data of partially edentulous patients (in the maxilla or mandible), of both genders, who were treated with implant therapy at São Leopoldo Mandic Institute and Research Center were selected consecutively. Patients with no need for bone augmentation and also patients with a need for horizontal bone augmentation, evaluated by CT scans performed prior to the beginning of treatment, were included. The implants should be functional for at least 1 year.

\section{Exclusion criteria}

Patients whose medical data included history of neoplastic disease treated with radiation therapy or chemotherapy, pregnant or breastfeeding women, those with systemic diseases or treatments affecting bone homeostasis, allergic reactions to components of the materials used, sinus pathologies, and smokers were excluded from the study. Patients subjected to vertical bone augmentation were also excluded from the retrospective analysis.

\section{Computed tomography (CT) analysis}

Imaging device

Cone beam images were acquired using the Sirona Galileos System (Sirona Dental Systems, Bensheim, Germany). This machine features a 14-second cycle at 220 degrees, which results in 200 individual exposures. Volume dimensions of $15 \times 15 \times 15 \mathrm{~cm}^{3}$ 
capture images at a high level of detail. The voxel size ranges from 0.15 to $0.30 \mathrm{~mm}$, and the grayscale is 12 bit. Image reconstruction time is approximately 4.5 minutes. The technology also allows for smallregion close-up views at double the detail without an additional scan.

\section{Image manipulation}

The images were evaluated using the Sidexis software (Sirona Dental Systems, Bensheim, Germany). This software allows viewing the different cuts at increasing magnifications for better accuracy. Alveolar ridge thickness and the presence or absence of cancellous bone were evaluated. All analyses were performed twice by a single experienced blinded examiner (AAP), with a 15-day interval between both analyses.

\section{Selection of biomaterial for each case}

As there is no consensus agreement in the scientific literature on how the biomaterial for horizontal bone augmentation should be chosen, all alternatives (autogenous, alloplastic, homogenous, and xenogenous) were given to the patients. However, the patients were told that the autogenous bone is the biological gold standard and, therefore, it could be more successful in more severe cases, besides the necessity (and morbidity) of a donor site. At the time of these grafts, the criterion of choice of the available types of grafts was the amount of graft required and how severe morbidity would be for bone harvest in the case of autogenous grafts. This way, the criteria used at that time involved donor site morbidity, the need to reduce the number of interventions, and the intention to use a material that was more widely available.

\section{Evaluation of bone grafting outcomes}

Bone grafting outcomes were retrospectively evaluated and categorized as satisfactory or unsatisfactory. When dental implants were placed without the need of a complementary bone graft procedure, the outcome was satisfactory. Conversely, if dental implant could not be placed without complementary bone grafting, the outcome was unsatisfactory.

\section{Results}

As shown in Table 1, out of 100 patients, only 17 $(17 \%)$ had no necessity for bone augmentation and only $8(8 \%)$ had severe atrophy, with no residual cancellous bone (Group A). On the other hand, 75 patients $(75 \%)$ required bone augmentation, but with the presence of some amount of remaining cancellous bone in the recipient bed (i.e., moderate atrophy) (Group P).

The outcomes of the bone augmentation procedures are described in Tables 2 and 3, and the descriptive analysis shows they were quite different between Group A and Group P. These outcomes demonstrated an unequivocal relationship between the success of the procedure - implantation of the dental implant - and the choice of the biomaterial, specially in Group A patients..

Table 1. Overview of patient population (need for bone augmentation).

\begin{tabular}{lc}
\hline Bone defect & $\%$ \\
\hline None & 17 \\
Severe & 8 \\
Moderate & 75 \\
\hline
\end{tabular}

Table 2. Overview of Group P patients.

\begin{tabular}{lccc}
\hline \multirow{2}{*}{ Biomaterial } & $\%$ & \multicolumn{2}{c}{ Outcomes (\%) } \\
\cline { 3 - 4 } & & Satisfactory & Unsatisfactory \\
\hline Alloplastic & 15.2 & 100 & 0 \\
Autogenous & 4.3 & 100 & 0 \\
Homogenous & 26.1 & 95.9 & 4.1 \\
Xenogenous & 35.9 & 97.0 & 3.0 \\
\hline
\end{tabular}

Satisfactory: dental implants placed without bone graft complement; Unsatisfactory: impossibility of dental implant placement without bone graft complement

Table 3. Overview of Group A patients.

\begin{tabular}{lccc}
\hline \multirow{2}{*}{ Biomaterial } & $\%$ & \multicolumn{2}{c}{ Outcomes (\%) } \\
\cline { 3 - 4 } & & Satisfactory & Unsatisfactory \\
\hline Autogenous & 25 & 100 & 0 \\
Homogenous & 37.5 & 0 & 100 \\
Xenogenous & 37.5 & 33.3 & 66.6 \\
\hline
\end{tabular}

Satisfactory: dental implants placed without bone graft complement; Unsatisfactory: impossibility of dental implant placement without bone graft complement 
Figure 1 illustrates the outcome of a graft procedure in a defect that had some amount of cancellous bone (Group P), treated with an allograft biomaterial. Figure 2 illustrates the outcome of a knife-edge bone defect (i.e., a defect with no residual cancellous bone) (Group A) also treated with an allograft biomaterial. It can be clearly noticed that in the situation where some amount of cancellous bone was present (Figure 1), reconstruction was more adequate than when cancellous bone was absent (Figure 2).

\section{Discussion}

The selected techniques and materials significantly affect the outcome of bone reconstruction procedures in terms of bone volume, quality, and amount of vital bone. The choices facing the dental surgeon at the time of extraction, ridge augmentation, or sinus grafts vary widely. When choosing a bone graft material, the surgeon should consider its ultimate effect on healing patterns in and around the native bone at the endpoint of the procedure. Moreover, the surgeon should consider the quality of the recipient bed and also have guidelines that could help him or her select the material for bone reconstruction.

The decision-making process for choosing an appropriate material for bone reconstruction should be based on the concept that the cortical lamellar bone does not have a high level of vitality, as stated by Lindhe and Lang. ${ }^{10}$ On the other hand, the cancellous compartment has high vitality and huge osteogenic potential, mainly due to the endosteum and to the bone marrow. ${ }^{11}$ Therefore, the presence of cancellous bone in the recipient bed, if exposed to perforations, could theoretically provide the osteoconductive biomaterial with some osteogenic

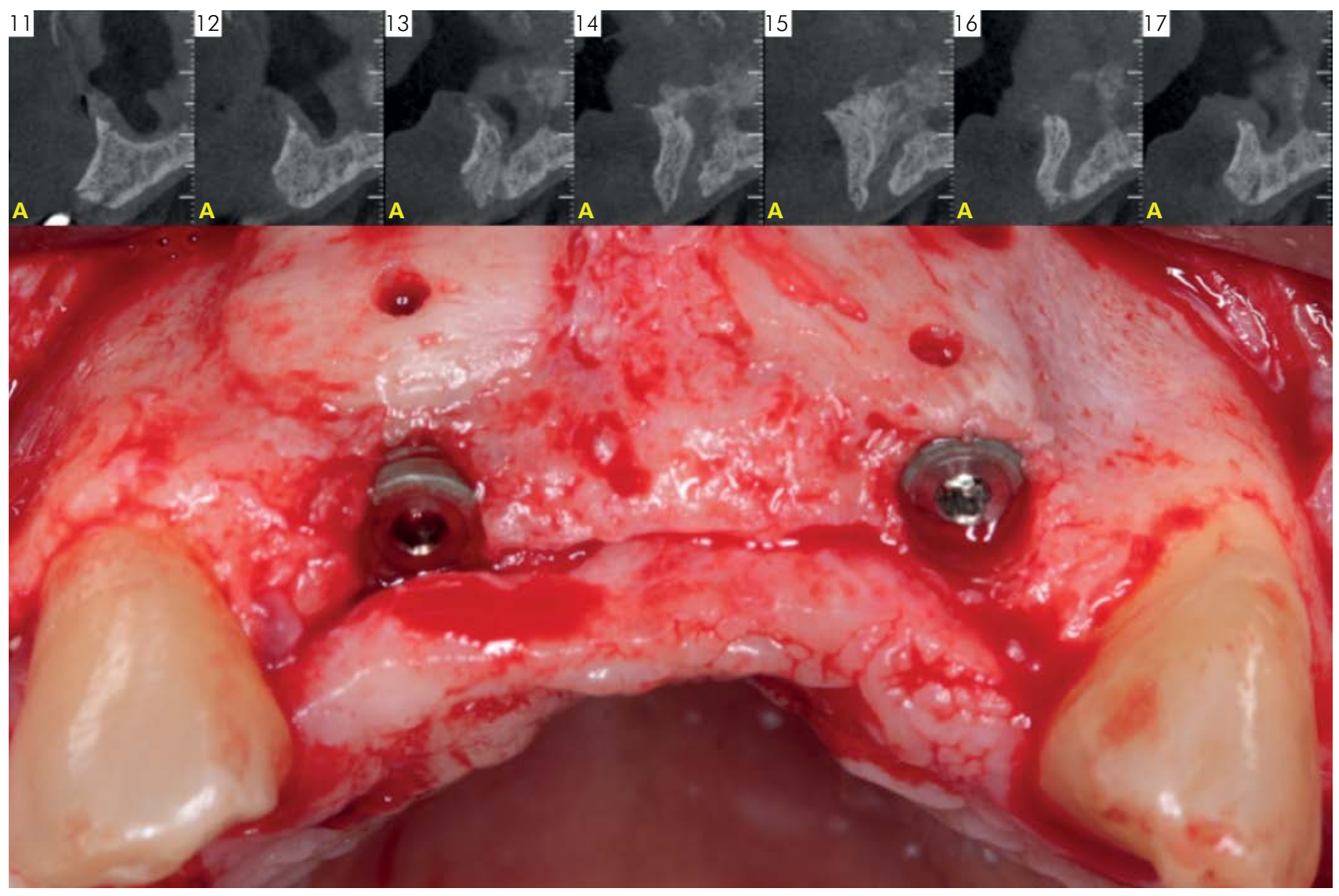

Figure 1. Preoperative tomographic view (parasagittal plane) and clinical view 6 months after horizontal augmentation with osteoconductive material. CT showing the presence of cancellous bone between the buccal and palatal cortical bone plates, despite important bone thickness loss (HAC 3). 


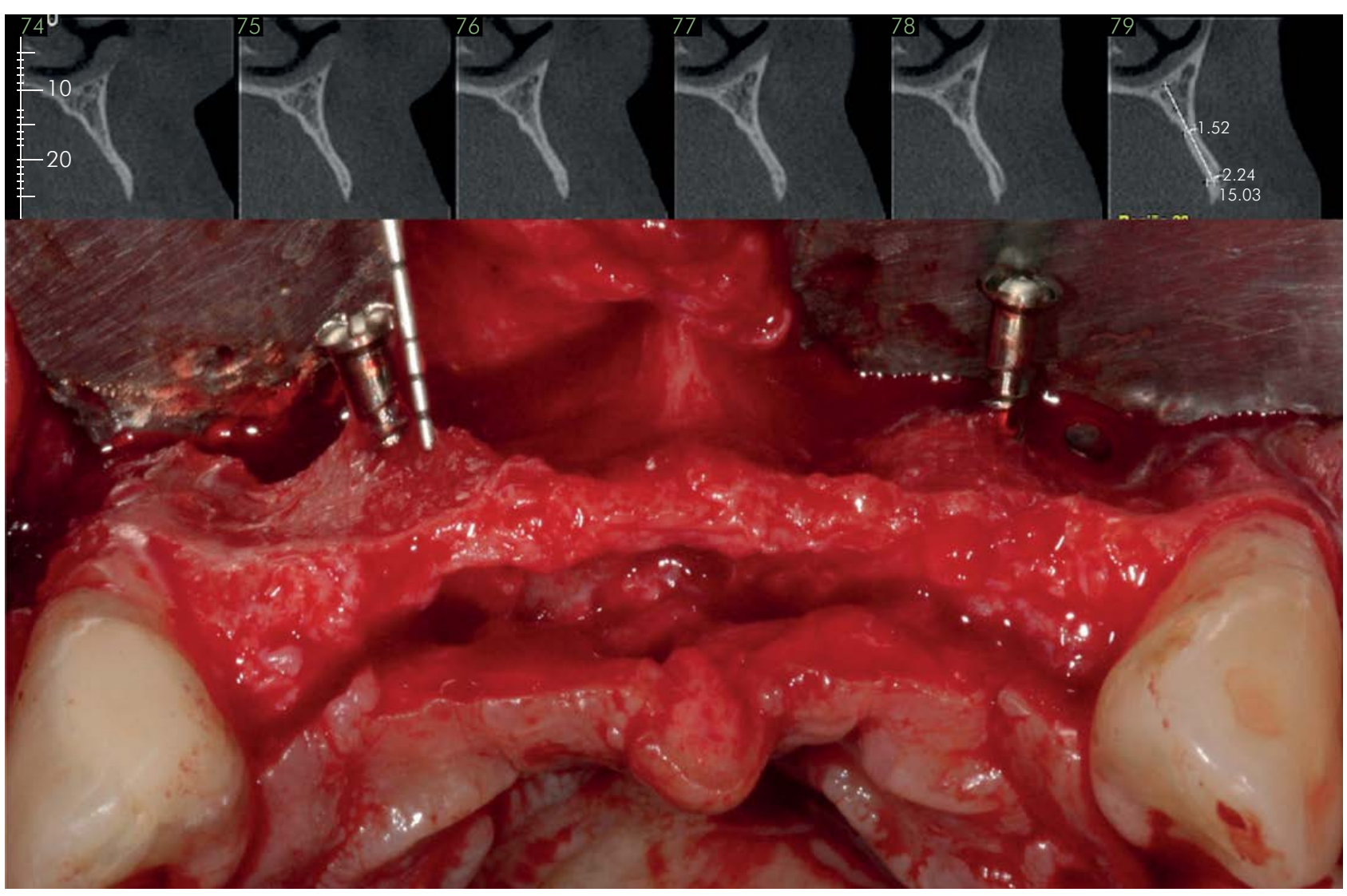

Figure 2. Preoperative tomographic view (parasagittal plane) and clinical view 6 months after horizontal augmentation with osteoconductive material. CT showing significant thickness bone loss and absence of cancellous bone between the buccal and palatal cortical bone plates in most of the alveolar bone (HAC 4).

cells to promote bone formation, as suggested by Gordh et al. ${ }^{12}$ These authors stated that cortical perforations induce the migration of the recipient bone marrow cells into the bone graft. By contrast, a knife-edge anatomy of an extremely resorbed bone, with no cancellous bone between the cortical plates, might not provide adequate cellularity to a nonvital osteoconductive biomaterial. So, cancellous bone evaluation by CT (especially in the parasagittal plane) should be considered for decision making in the bone regeneration approach, especially when the use of bone substitutes (i.e., allogenous, xenogenous, and synthetic biomaterials) is being considered. Therefore, a classification that could allocate patients to categories based on initial CT findings may be of major importance in the bone reconstruction field. This necessity encouraged the authors of this paper to propose a classification of horizontal alveolar changes (HAC).
HAC classification was idealized by the authors of this article according to horizontal bone defects, both in the maxilla and mandible; thus, it does not include height defects that demand a vertical bone reconstruction. $\mathrm{HAC}$ is divided into four main categories defined according to the morphological aspect of residual bone, evaluated by CT images. Therefore, the following HAC classification was proposed:

a. HAC1: slight bone resorption with no need for bone reconstruction due to the possibility of implant placement in an ideal position without grafting. There is presence of cancellous bone between the cortical buccal and palatal/lingual bone plates and the possibility of a single surgical approach, with immediate implant placement.

b. HAC 2: slight bone resorption with minor need for bone reconstruction, which can be done with the use of an osteoconductive biomaterial because of the presence of cancellous bone 
between the cortical buccal and palatal/lingual bone plates. It also allows for a single surgical approach, with immediate implant placement.

c. HAC 3: moderate bone resorption, but still with remaining cancellous bone at the residual alveolar site, requiring just the use of an osteoconductive biomaterial for reconstruction. However, as the required bone reconstruction is significant, in these situations a two-stage surgical approach is usually needed: firstly just the bone graft procedure and, a few months later, the implant.

d. HAC 4: severe bone resorption, with no remaining cancellous bone at the residual alveolar site, requiring the use of an osteoconductive, osteoinductive, and osteogenic material (i.e., autogenous bone graft or bone tissue engineering with live cell transplant or bone inductive proteins). As the required bone reconstruction is significant, a two-stage surgical approach is imperative. Table 4 summarizes the HAC classification.

As shown in Table 4, it would be of major importance - in addition to the understanding of the recipient bed characteristic - the recognition of different graft materials potential. In this respect, the potentials are classically divided into three categories: a) osteoconduction; b) osteoinduction, and c) osteogenesis. However, a material can theoretically have only one, two, or even three potentials simultaneously. The osteoconductive material shows revascularization capability with consequent diapedesis, allowing for migration and cell adhesion. Therefore, the osteoconductive material depends entirely on the recipient bed cellularity. The osteoinductive material has bioactive molecules that favor cell differentiation. The osteogenic material has bone cells with the potential of forming bone per se. The material with osteogenic characteristics contains osteoblasts (differentiated cells) and/or mesenchymal stem cells (undifferentiated cells with osteogenic differentiation capacity), which secrete osteoinductive growth factors and deposit the necessary bone matrix..$^{13}$

Therefore, in really critical bone reconstruction situations, without some amount of cancellous bone at the recipient bed (i.e., HAC 4), it is imperative that a graft with osteogenic capacity be used, since the recipient bed is not capable of providing adequate vascularization and, consequently, osteoprogenitor cells. Therefore, HAC 4 defects are not likely to be built just with bone substitute biomaterials, since these are, in their vast majority, exclusively osteoconductors. Keeping this in mind, it might be concluded that HAC 4 defects should

Table 4. Horizontal Alveolar Change (HAC) classification.

\begin{tabular}{|c|c|c|c|c|c|c|c|}
\hline Classification & $\begin{array}{l}\text { Amount of } \\
\text { alveolar } \\
\text { horizontal loss }\end{array}$ & $\begin{array}{l}\text { Surgical } \\
\text { approach } \\
\text { (stages) }\end{array}$ & $\begin{array}{l}\text { Presence of } \\
\text { cancellous } \\
\text { bone }\end{array}$ & $\begin{array}{l}\text { Imperative need of autogenous } \\
\text { graft or bone inductive } \\
\text { proteins/live cell transplant }\end{array}$ & $\begin{array}{l}\text { Material for } \\
\text { augmentation }\end{array}$ & $\begin{array}{l}\text { Maxilla } \\
\text { aspect on } \\
\text { CT }\end{array}$ & $\begin{array}{l}\text { Mandible } \\
\text { aspect on } \\
\text { CT }\end{array}$ \\
\hline $\mathrm{HACl}$ & Small & Single & Yes & No & No & & \\
\hline HAC2 & Small & Single & Yes & No & $\begin{array}{l}\text { Osseoconductive } \\
\text { biomatrial }\end{array}$ & & \\
\hline HAC3 & Moderate & Two & Yes & No & $\begin{array}{l}\text { Osseoconductive } \\
\text { biomatrial }\end{array}$ & & \\
\hline $\mathrm{HAC} 4$ & Large & Two & No & Yes & $\begin{array}{l}\text { Autogenous or } \\
\text { biomaterial with bone } \\
\text { inductive proteins/live } \\
\text { cell transplant }\end{array}$ & & \\
\hline
\end{tabular}


be primarily treated by autogenous bone graft, as shown in Table 3, where the use of autogenous bone resulted in $100 \%$ of satisfactory outcomes (compared to $33.3 \%$ and $0.0 \%$ obtained with xenogenous and homogenous bones, respectively). However, morbidity and complications related to the donor site may raise doubts among many patients with this type of defect when selecting implantsupported rehabilitation. This allows for the use of bone tissue engineering concepts, especially through the use of cell therapy in which the bone marrow is the tissue source, since the osteogenic potential is directly related to the presence of osteoprogenitor cells. Accordingly, more than 40 years ago, Friedenstein et al. ${ }^{14}$ identified a cell population with high osteogenic potential in a cell suspension of bone marrow, setting the path for bone tissue engineering with the use of bone marrow cells, as shown by Aloise et al., ${ }^{15}$ Oliveira et al. ${ }^{16}$ Pelegrine et al., ${ }^{17}$ and Corrêa et al..$^{18}$

As horizontal bone defects HAC 2 and HAC 3 do not require an autograft material (Table 2), the first option could be an osteoconductive biomaterial, such as a xenograft, an allograft, or even a synthetic material, which eventually led to a good level of satisfactory outcomes in this retrospective study $(97.0 \%$,

\section{References}

1. Buser D, Sennerby L, De Bruyn H. Modern implant dentistry based on osseointegration: 50 years of progress, current trends and open questions. Periodontol 2000. 2017 Feb;73(1):7-21. https://doi.org/10.1111/prd.12185

2. Buser $D$, Brägger $U$, Lang NP, Nyman $S$. Regeneration and enlargement of jaw bone using guided tissue regeneration. Clin Oral Implants Res. 1990 Dec;1(1):22-32. https://doi.org/10.1034/j.1600-0501.1990.010104.x

3. Jensen SS, Terheyden $\mathrm{H}$. Bone augmentation procedures in localized defects in the alveolar ridge: clinical results with different bone grafts and bone-substitute materials. Int J Oral Maxillofac Implants. 2009;24 Suppl:218-36.

4. Sakkas A, Schramm A, Winter K, Wilde F. Risk factors for postoperative complications after procedures for autologous bone augmentation from different donor sites. J Craniomaxillofac Surg. 2018 Feb;46(2):313-22. https://doi.org/10.1016/i.jcms.2017.11.016

5. Costa CE, Pelegrine AA, Fagundes DJ, Simoes MJ, Taha MO. Use of corticocancellous allogeneic bone blocks impregnated with bone marrow aspirate: a clinical, tomographic, and histomorphometric study. Gen Dent. 2011 Sep-Oct;59(5):e200-5.
$95.9 \%$, and $100 \%$, respectively). Using an autograft in these situations might be considered an overtreatment because there is no need for a second surgical site approach (donor site). On the other hand, the use of an osteoconductive biomaterial in HAC 4 defects would probably result in failure. That is why the authors of this article state that an osteoconductive biomaterial can be used up to HAC 3, but not in HAC 4 (as observed in Figures 1 and 2).

Therefore, the goal of this paper was to open up a discussion about the need to establish bone augmentation guidelines and to suggest a possible classification, which may be corroborated in a clinical trial.

\section{Conclusion}

Based on this retrospective study, the presence of cancellous bone might be predictive and predictable when using bone substitutes. In case of absence of cancellous bone in the recipient bed, the use of a vitalized graft, such as an autogenous bone, seems to be mandatory. Some guidelines were presented in this study, but their applicability must be confirmed in future investigations.

6. Araujo DB, de Jesus Campos E, Oliveira MA, Lima MJ, Martins GB, Araujo RP. Surgical elevation of bilateral maxillary sinus floor with a combination of autogenous bone and lyophilized bovine bone. J Contemp Dent Pract. 2013 May; 14(3):445-50. https://doi.org/10.5005/ip-journals-10024-1342

7. Dottore AM, Kawakami PY, Bechara K, Rodrigues JA, Cassoni A, Figueiredo LC et al. Stability of implants placed in augmented posterior mandible after alveolar osteotomy using resorbable nonceramic hydroxyapatite or intraoral autogenous bone: 12-month follow-up. Clin Implant Dent Relat Res. 2014 Jun;16(3):330-6. https://doi.org/10.1111/cid.12010

8. Pang C, Ding Y, Zhou H, Qin R, Hou R, Zhang G et al. Alveolar ridge preservation with deproteinized bovine bone graft and collagen membrane and delayed implants. J Craniofac Surg. 2014 Sep;25(5):1698-702. https://doi.org/10.1097/SCS.0000000000000887

9. Aghaloo TL, Hadaya D. Basic principles of bioengineering and regeneration. Oral Maxillofac Surg Clin North Am. 2017 Feb;29(1):1-7. https://doi.org/10.1016/i.coms.2016.08.008 
Horizontal Bone Reconstruction on sites with different amounts of native bone: a retrospective study

10. Lindhe J, Lang NP. Clinical periodontology and implant dentistry. 6th ed. Wiley-Blackwell; 2015.

11. Gray JC, Elves MW. Early osteogenesis in compact bone isografts: a quantitative study of contributions of the different graft cells. Calcif Tissue Int. 1979;29(3):225-37. https://doi.org/10.1007/BF02408085

12. Gordh M, Alberius $P$, Lindberg L, Johnell $O$. Bone graft incorporation after cortical perforations of the host bed. Otolaryngol Head Neck Surg. 1997 Dec;117(6):664-70. https://doi.org/10.1016/S0194-5998(97)70050-0

13. Sundelacruz S, Kaplan DL. Stem cell- and scaffold-based tissue engineering approaches to osteochondral regenerative medicine. Semin Cell Dev Biol. 2009 Aug;20(6):646-55. https://doi.org/10.1016/j.semcdb.2009.03.017

14. Friedenstein AJ, Petrakova KV, Kurolesova AI, Frolova GP. Heterotopic of bone marrow. Analysis of precursor cells for osteogenic and hematopoietic tissues.

Transplantation. 1968 Mar;6(2):230-47. https://doi.org/10.1097/00007890-196803000-00009

15. Aloise AC, Pelegrine AA, Zimmermann A, Mello E Oliveira $R$, Ferreira LM. Repair of critical-size bone defects using bone marrow stem cells or autogenous bone with or without collagen membrane: a histomorphometric study in rabbit calvaria. Int J Oral Maxillofac Implants. 2015 Jan-Feb;30(1):208-15. https://doi.org/10.11607/jomi.4010

16. Oliveira TA, Aloise AC, Orosz JE, Mello E Oliveira R, Carvalho P, Pelegrine AA. Double centrifugation versus single centrifugation of bone marrow aspirate concentrate in sinus floor elevation: a pilot study. Int J Oral Maxillofac Implants. 2016 JanFeb;31(1):216-22. https://doi.org/10.11607/jomi.4170 17. Pelegrine AA, Teixeira ML, Sperandio M, Almada TS, Kahnberg KE, Pasquali PJ et al. Can bone marrow aspirate concentrate change the mineralization pattern of the anterior maxilla treated with xenografts? A preliminary study. Contemp Clin Dent. 2016 Jan-Mar;7(1):21-6. https://doi.org/10.4103/0976-237X.177112

18. Corrêa SCL, Sousa JE, Pasquali PJ, Macedo LGS, Aloise AC, Teixeira ML et al. Use of bone allograft with or without bone marrow aspirate concentrate in appositional reconstructions: a tomographic and histomorphometric study. Implant Dent. 2017 Dec;26(6):915-21. https://doi.org/10.1097/ID.0000000000000669 